\title{
Protocol for AREST: Apixaban for Early Prevention of Recurrent Embolic Stroke and Hemorrhagic Transformation-A Randomized Controlled Trial of Early Anticoagulation After Acute Ischemic Stroke in Atrial Fibrillation
}

\section{OPEN ACCESS}

Edited by:

Laszlo Csiba,

University of Debrecen, Hungary

Reviewed by:

Henry Ma,

Monash University, Australia Richard Zweifler,

Ochsner Health System, United States

*Correspondence:

David Z. Rose

drose1@health.usf.edu

Specialty section: This article was submitted to

Stroke,

a section of the journal

Frontiers in Neurology

Received: 13 June 2019

Accepted: 27 August 2019

Published: 20 September 2019

Citation:

Rose DZ, Meriwether JN, Fradley MG, Renati S, Martin RC, Kasprowicz T,

Patel A, Mokin M, Murtagh R, Kip K, Bozeman AC, McTigue T, Hilker N, Kirby B, Wick N, Tran N, Burgin WS and Labovitz AJ (2019) Protocol for AREST: Apixaban for Early Prevention

of Recurrent Embolic Stroke and

Hemorrhagic Transformation $-A$ Randomized Controlled Trial of Early Anticoagulation After Acute Ischemic Stroke in Atrial Fibrillation.

Front. Neurol. 10:975.

doi: 10.3389/fneur.2019.00975
David Z. Rose ${ }^{1 *}$, John N. Meriwether ${ }^{2}$, Michael G. Fradley ${ }^{2}$, Swetha Renati ${ }^{1}$, Ryan C. Martin ${ }^{2}$, Thomas Kasprowicz ${ }^{2}$, Aarti Patel ${ }^{2}$, Maxim Mokin ${ }^{1}$, Ryan Murtagh ${ }^{3}$, Kevin Kip ${ }^{4}$, Andrea C. Bozeman ${ }^{1}$, Tara McTigue ${ }^{1}$, Nicholas Hilker ${ }^{1}$, Bonnie Kirby ${ }^{2}$, Natasha Wick ${ }^{2}$, Nhi Tran ${ }^{2}$, W. Scott Burgin ${ }^{1}$ and Arthur J. Labovitz ${ }^{2}$

\footnotetext{
${ }^{1}$ Department of Neurology, Morsani College of Medicine, University of South Florida, Tampa, FL, United States, ${ }^{2}$ Department of Cardiology, Morsani College of Medicine, University of South Florida, Tampa, FL, United States, ${ }^{3}$ Department of Radiology, Morsani College of Medicine, University of South Florida, Tampa, FL, United States, ${ }^{4}$ College of Public Health, University of South Florida, Tampa, FL, United States
}

Background: Optimal timing to initiate anticoagulation after acute ischemic stroke (AIS) from atrial fibrillation (AF) is currently unknown. Compared to other stroke etiologies, AF typically provokes larger infarct volumes and greater concern of hemorrhagic transformation, so seminal randomized trials waited weeks to months to begin anticoagulation after initial stroke. Subsequent data are limited and non-randomized. Guidelines suggest anticoagulation initiation windows between 3 and 14 days post-stroke, with Class lla recommendations, and level of evidence B in the USA and C in Europe.

Aims: This open-label, parallel-group, multi-center, randomized controlled trial AREST (Apixaban for Early Prevention of Recurrent Embolic Stroke and Hemorrhagic Transformation) is designed to evaluate the safety and efficacy of early anticoagulation, based on stroke size, secondary prevention of ischemic stroke, and risks of subsequent hemorrhagic transformation.

Methods: Subjects are randomly assigned in a 1:1 ratio to receive early apixaban at day 0-3 for transient ischemic attack (TIA), 3-5 for small-sized AIS $(<1.5 \mathrm{~cm})$, and $7-9$ for medium-sized AIS ( $1.5 \mathrm{~cm}$ or greater but less than a full cortical territory), or warfarin at 1 week post-TIA or 2 weeks post-stroke. Large AISs are excluded.

Study Outcomes: Primary: recurrent ischemic stroke, TIA, and fatal stroke; secondary: intracranial hemorrhage $(\mathrm{ICH})$; hemorrhagic transformation $(\mathrm{HT})$ of ischemic stroke; cerebral microbleeds (CMBs); neurologic disability [e.g., modified Rankin Scores (mRS), National Institutes of Health Stroke Scale (NIHSS), Stroke Specific Quality of Life scale (SS-QOL)]; and cardiac biomarkers [e.g., AF burden, transthoracic echo (TTE)/transesophageal echo (TEE) abnormalities]. 
Sample Size Estimates: Enrollment goal was 120 for 80\% power (two-sided type I error rate of 0.05 ) to detect an absolute risk reduction of $16.5 \%$ postulated to occur with apixaban in the primary composite outcome of fatal stroke/recurrent ischemic stroke/TIA within 180 days. Enrollment was suspended at 91 subjects in 2019 after a focused guideline update recommended direct oral anticoagulants (DOACs) over warfarin in AF, excepting valvular disease (Class I, level of evidence A).

Discussion: AREST will offer randomized controlled trial data about timeliness and safety of anticoagulation in AIS patients with AF.

Clinical Trial Registration: www.ClinicalTrials.gov, identifier NCT02283294.

Keywords: acute ischemic stroke, atrial fibrillation, anticoagulation timing, direct oral anticoagulant, apixaban, transient ischemic attack

\section{INTRODUCTION, BACKGROUND, AND AIMS}

For patients with acute ischemic stroke (AIS) due to atrial fibrillation (AF), it is currently unknown when the optimal time is to prescribe oral anticoagulation. Early use may prevent secondary ischemic stroke from AF but may provoke hemorrhagic transformation (HT). Later use may prevent HT, but unanticoagulated AF can provoke further AIS, particularly in patients at higher risk based on CHADSVASc score. Early initiation of oral anticoagulation may mitigate the exorbitant fivefold recurrent risk of AIS seen in AF (when compared to other stroke etiologies) - a risk that is especially highest within the first few weeks post-stroke (1-3). Although early anticoagulation may result in fewer ischemic events, the much-feared potential harm (i.e., bleeding) counterbalancing this benefit has never been formally evaluated in a randomized, clinically controlled manner and may be overestimated.

Typically, AF also results in greater infarct volumes, again compared to other etiologies of stroke. Hence, use of early anticoagulation upon an unstable, fragile, neuronal infarct bed poses a potentially greater risk of HT than waiting a few days (or weeks) for it to mature. Unsurprisingly, HT itself results in increased morbidity and mortality via mass effect, cerebral edema, and herniation syndromes, which can be fatal $(1,2)$.

Treatment guidelines have emerged without much firm evidence on this topic and only offer vague suggestions for timing of anticoagulation initiation in AF-related AIS. The American College of Chest Physicians (2012) and the American Heart/Stroke Association (2014) propose initiation within 2 weeks of a cardioembolic stroke, except in cases of large strokes, uncontrolled hypertension, or bleeding conditions $(4,5)$. The European Society of Cardiology guidelines (2016) are slightly less nebulous, with a marginally more specific window of 312 days, except for a list of highest-risk individuals similar to the other associations' suggestions (6). Consensus, rather than any randomized prospective data, produced these Class IIa recommendations, with level of evidence B in the USA and C in Europe.

Historically, opinion about oral anticoagulation timing for AF-related AIS has been based on experience with warfarin. With the interval development of better-tolerated direct oral anticoagulant (DOAC) therapy, which takes only a few hours to reach therapeutic level in the blood, not days like warfarin does, the optimal timing of anticoagulation initiation after an ischemic stroke is even more unclear-yet even more necessary given the rapid adoption of the DOAC class for primary and secondary stroke prevention from AF. Non-randomized and openly confounded studies have inferred safety and tolerability of early DOAC use post-stroke from AF (7-11); however uncertainty exists about the actual risk-benefit ratio and the appropriate strategic latency required before anticoagulation initiation in these fragile patients.

Given the clinical equipoise in this at-risk population, and to address this grossly unmet need, the neurology and cardiology departments at the University of South Florida (vis-à-vis our "USF Neuro-Cardiac Program") designed the randomized, controlled AREST trial: Apixaban for Early Prevention of Recurrent Embolic Stroke and Hemorrhagic Transformation. We hypothesize that in patients with non-valvular AF and AIS, initiating early oral anticoagulation with the DOAC apixaban (precise timing based on actual stroke size) results in fewer recurrent ischemic strokes or transient ischemic attacks (TIAs), without significantly increasing the risk of intracranial hemorrhage $(\mathrm{ICH})$, vs. warfarin initiated at 14 days (9-11).

\section{METHODS}

\section{Design}

AREST is an open-label, parallel-group, randomized, active controlled trial designed to examine the effect of early initiation of the DOAC apixaban in AF at day 0-3 for TIA, 3-5 for small-sized AIS, and 7-9 for medium-sized AIS, vs. warfarin at 1 week post-TIA or 2 weeks post-stroke. Figure 1 shows the study flowchart. Subjects are prospectively identified, give consent, and are enrolled by the NeuroCardiac Program team at the University of South Florida in Tampa, with additional sites including Bayfront Health in St. Petersburg, University of Louisville in Kentucky, and University of California-Los Angeles. The diagnosis of AIS, $\mathrm{ICH}, \mathrm{HT}$, and cerebral microbleed (CMB) was adjudicated by committee; imaging discrepancies were resolved by investigator consensus. 


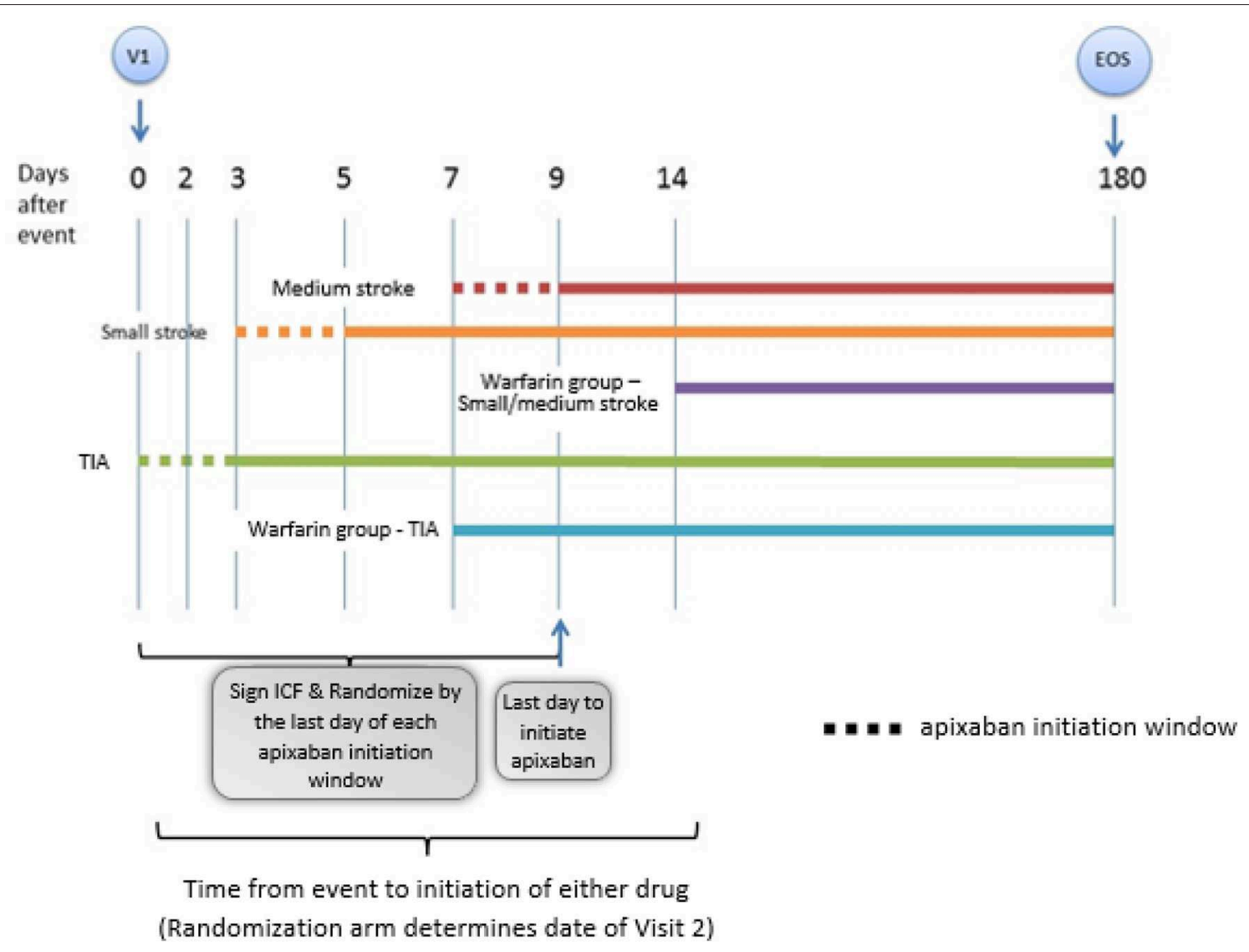

FIGURE 1 | This is a representation of the general study schema for the AREST (Apixaban for Early Prevention of Recurrent Embolic Stroke and Hemorrhagic Transformation) study (V1 = visit 1, time 0; EOS = end of study, day 180). Details of the follow up visits entailed in the follow up section of the proof of concept.

\section{Patient Population, Inclusion, and Exclusion Criteria}

Eligible patients are those $\geq 18$ years of age who have experienced AIS within 3-5 days, or TIA within 3 days, and have AF by history, electrocardiogram (EKG), telemetry, or longer-term cardiac monitor. Electrographic criteria include irregular atrial activity without distinct regular recurring $\mathrm{P}$-wave and an irregularly irregular R-to-R interval if atrial-to-ventricular conduction is intact. Mode switch episodes recorded by implantable pacemakers and defibrillators were insufficient to diagnose $\mathrm{AF}$, if unaccompanied by corresponding intracardiac electrograms. An electrophysiologist reviewed recorded arrhythmias and confirmed AF. Although neuroimaging in some potential subjects may have suggested cardioemboli, AREST enrolled neither cryptogenic stroke nor embolic stroke of undetermined source (ESUS). Generally, without cardiac monitoring, there may be an overdiagnosis of cryptogenic strokes and underdiagnosis of AF (12); however, we permitted only a clear and convincing past medical history of $\mathrm{AF}$ or a newly identified $\mathrm{AF}$ rhythm pattern for study entry.

Strokes were defined by the presence of focal neurological deficits and classified by neuroradiographic size of the largest area of AIS. Neuroimaging utilized diffusion-weighted image (DWI) sequences of brain magnetic resonance imaging (MRI), or head computed tomography (CT) if the patient was MRIincompatible (pacemaker, claustrophobia, etc.). By definition, TIA must have resolved focal deficits without acute DWI changes on MRI or hypodensities on CT. The clinical classification of AIS was based on the Oxfordshire Community Stroke Project (OCSP) for predicting the site and size of AIS on imaging and has previously been validated (13). Small-sized AIS can involve the anterior or posterior circulation and is $<1.5-\mathrm{cm}$ in largest dimension. Medium-sized AIS is $\geq 1.5 \mathrm{~cm}$ but less than a full cortical territory of the anterior cerebral artery (ACA), middle cerebral artery (MCA), or posterior cerebral artery (PCA). Largesized AIS (complete arterial territory) and brainstem strokes are excluded due to potentially excessive (and fatal) HT risk from early anticoagulation.

Other exclusion criteria were primary ICH, HT of the AIS, or AIS from other etiologies (i.e., ipsilateral carotid stenosis). Subjects could be enrolled with $\leq 3 \mathrm{CMBs}$ on gradient recovery echo (GRE) sequence on MRI-or susceptibility weighted imaging (SWI) if GRE is unavailable. Subjects were excluded if MRI detected CMBs within or adjacent to the infarct, or $>3$ CMBs anywhere in the brain (cortical or subcortical); these 
restrictions were created to avoid the potentially higher risk of $\mathrm{CMB}$ expansion when starting anticoagulation.

Further exclusion criteria include therapeutic anticoagulation at the time of admission [active warfarin use with admission International Normalized Ratio (INR) $\geq 2.0$ or administered two consecutive doses of DOAC] or another need for anticoagulation [e.g., mechanical valve, Deep Vein Thrombosis (DVT), hypercoagulable state] or dual anti-platelet therapy (e.g., cardiac stent); major bleeding within the last 6 months; blood dyscrasias; traumatic brain injury (TBI)-associated ICH within 1 year; blood pressure $\geq 180 / 100 \mathrm{~mm} \mathrm{Hg}$ on day of randomization [per Principal Investigator (PI) discretion]; illicit drug and/or alcohol use (per PI discretion); liver disease (defined as liver enzymes twice the upper limit); end stage renal disease; concurrent use of specific dual inhibitors of CYP3A4 and P-glycoprotein; anemia with hemoglobin $<9$ $\mathrm{gm} / \mathrm{dl}$; thrombocytopenia with platelet count $<100 \mathrm{~K} / \mu \mathrm{l}$; or pregnant/lactating women (must have a negative pregnancy test prior to enrollment).

\section{Randomization and Intervention}

Subjects are randomly assigned in a 1:1 ratio to either apixaban or warfarin. Prior to discharge, subjects receive neuroimaging, arterial studies of the head and neck, and transthoracic echo (TTE) with or without transesophageal echo (TEE). A 30-day cardiac event monitor may be given at discharge or afterwards. Subjects are followed at the following intervals: post-stroke/TIA days $14,30,60,90,120,150$, and 180 . An end-of-study phone interview occurs at day 210. At follow-up visits, subjects receive vitals, intermittent history, EKG, event monitor review, physical examination, NIH Stroke Scale (NIHSS), modified Rankin Scores (mRS), compliance assessment (medication review), and adverse event assessment. At baseline, days 30 and 180 (or early withdrawal) visits, a Stroke Specific Quality of Life scale (SSQOL) is collected. At baseline and days 30, 90, and 180, labs are collected (lipid panel, coagulation studies, comprehensive metabolic panel, and complete blood count). MRI (or CT if MRIincompatible) is performed at baseline and days 14 and 180 (or early withdrawal).

\section{Study Outcomes}

Primary end points include a composite of recurrent ischemic stroke, TIA, and fatal stroke at days 30 and 180 (primary efficacy outcome). Secondary end points are ICH (primary safety end point), which can include primary hemorrhagic stroke, HT of ischemic stroke, or new CMB on GRE or SWI. Follow-up continues until day 180 from the index stroke event or until one of the primary or secondary end points is reached-at which time the subject completes the study. Additional outcome data include mRS and NIHSS for Neurologic Disability, SS-QOL, TTE/TEE abnormalities, and AF burden on cardiac event monitoring.

\section{Sample Size Estimates}

The enrollment goal was 120 patients in order to provide $80 \%$ power (two-sided type I error rate of 0.05 ) to detect a large absolute risk reduction of $16.5 \%$ postulated to occur with apixaban in the primary composite outcome of fatal stroke/recurrent ischemic stroke/TIA at 180 days. Enrollment was suspended at 91 subjects in early 2019 after a focused guideline update recommended DOACs over warfarin in AF, excepting valvular disease (Class I, level of evidence A).

\section{Statistical Analyses}

For the primary composite end point, the proportion of patients experiencing the outcome will be computed along with the absolute risk difference by random assignment and corresponding 95\% confidence interval. Similarly, the number needed to treat (NNT) and 95\% confidence interval will be calculated based on the incidence rates of the primary composite outcome in the two treatment groups. Because random assignment is stratified by stroke severity (small stroke, medium stroke, TIA), balanced distribution is expected. Given the modest sample size, potential unexpected confounding of baseline covariates (imbalanced distributions by random assignment) will be assessed by the use of Student $t$-tests or Wilcoxon tests for continuous variables and chi-square tests for categorical variables, conservatively using a $p$-value of $<0.15$. A general log-linear model will be fit with clinical site as a random effect, random assignment as the primary predictor (main effect) of interest, adjustment for any potential confounders, and the primary composite end point as the outcome variable. This will result in an adjusted risk ratio and 95\% confidence interval for apixaban vs. warfarin. Separate models will be fit at 30 and 180 days. The intent-to-treat principle will be used in all analyses. For assessment of safety, the incidence of $\mathrm{ICH}$ will be compared by random assignment.

\section{Compliance With Ethical Standards}

AREST has been approved by the Institutional Review Board at the University of South Florida Morsani College of Medicine and at Tampa General Hospital as well as all participating study hospitals. All study procedures are in accordance with the provisions of the International Conference of Harmonization Good Clinical Practice and the 1964 Declaration of Helsinki and its later amendments. Written informed consent is obtained from each patient (or proxy) before enrollment. AREST is registered on ClinicalTrials.gov (NCT02283294).

\section{Safety and Data Monitoring Body}

No safety issues are expected. Any serious study-related events, along with the events captured as secondary study outcomes, will be reported to the independent Data Safety Monitoring Board (DSMB) committee.

\section{Study Organization and Funding}

AREST is an initiative of the University of South Florida Morsani College of Medicine, in conjunction with the University of Louisville in Kentucky; Bayfront Health in St. Petersburg, Florida; and the University of California-Los Angeles. This randomized study is funded by the Bristol Myers Squibb/Pfizer Alliance.

\section{COLLABORATION}

AREST was the brainchild of the academic vascular neurology and cardiology services at the University of South Florida (USF) 
Morsani College of Medicine. In collaboration with our 1,018bed teaching facility, Tampa General Hospital, USF established a Neuro-Cardio Program (NCP) to create trials such as AREST and to address mutual patients' unmet needs. Starting NCP was a long sought-after effort with three major goals: (1) coidentification and co-enrollment of subjects into clinical trials that may benefit both specialties; (2) co-consultation on patients with overlapping diagnoses in a Thursday clinic with "freezeand-thaw" time slots for mixed use; and (3) Tuesday radiology conferences for assessment of brain MRIs for CMB, HT, or other neuro-abnormalities, and echocardiograms for thrombi, valvular disease, or other cardio-abnormalities. We have found that interdisciplinary interaction at USF has led to optimal decisions on cases of neurocardiogenic syncope, insertion of implantable loop recorders or telemetry patches looking for $\mathrm{AF}$ in cryptogenic stroke, closure of patent foramen ovale (PFO) for paradoxical embolic ischemic stroke, AF treatment in patients unsuitable for long-term anticoagulation and shared decision-making (SDM) for left atrial appendage closure device implantation. At our monthly NCP logistical/planning meetings, we review treatment algorithms and protocols for our mutual patient population, assess progress of our clinical trials and database collection, and fix any inpatient/outpatient comanagement obstacles. Without NCP, local investigator-initiated trials such as AREST are more difficult to foster, design, fund, and complete.

\section{DISCUSSION}

The late, great, centenarian neurologist Dr. C. Miller Fisher once quipped, "If you are treating even one patient in your practice with anticoagulation, you should wake up once a week or so in a cold sweat" (14).

For decades, the infamous vitamin-K antagonist (VKA) warfarin has been the cornerstone of stroke prevention in AF; however, the panoply of drawbacks of VKA make it cumbersome to take and manage $(15,16)$. Regardless, VKA is still abundantly prescribed for AF in the United States and around the world, mostly due to its inexpensive direct cost. DOACs are priced higher but may result in a significantly shorter length of stay and decreased overall and indirect medical costs compared to warfarin (17). Moreover, DOACs have shown non-inferiority (or in some cases superiority) to VKA in ischemic stroke prevention for AF as well as significantly less bleeding, especially in the intracranial bed $(15,16,18$, 19). The initial DOAC-vs.-warfarin randomized controlled trials for stroke prevention in AF, published between 2009 and 2013, did not test early use of either the study drug or its comparator, so any advantage in ischemic stroke risk reduction (or bleeding risk) during this initial time period remains unknown $(7,18-20)$.

These seminal clinical trials delayed anticoagulation weeks to months post-stroke, presumably due to a perceived higher HT risk vs. any potential benefit of very early secondary stroke prevention from either VKA or DOAC $(15,16,18$, 19). Undoubtedly, however, treatment initiation delay has consequence: about $10 \%$ of patients with AF-related stroke do experience recurrent embolic events within the first 30 days $(7,8,19,20)$, with immediate ( $<2$ weeks) risk ranging from 0.1 to $1.3 \%$ per day (9). This "threshold delay," described in 2015 by the VISTA (Virtual International Stroke Trials Archive) collaborators, represents the time point after AF-stroke when the value of anticoagulation switches from neutral (or even harmful) to beneficial (8). Prospective observational and non-randomized cohort data suggest that early initiation of anticoagulation may reduce embolic risk between 4 and 14 days post-stroke (9) and that anticoagulation started 2-3 days post-stroke, generally, could be ideal (8). In 2016, a small, prospective, open-label, MRI study of 60 patients with AF treated early (median of 3 days) with the DOAC rivaroxaban after TIA or small- to medium-sized stroke (median NIHSS of 2), identified three new HT and five HT progressions, all reportedly asymptomatic (7). Larger ischemic strokes, however, pose greater fear for HT with anticoagulation, as multivariate analysis revealed an association of higher rates of symptomatic ICH as well as AIS recurrence, interestingly (9).

Current clinical practice is variable for starting anticoagulation in AIS with AF. Guidelines offer only Class IIa recommendations, wide-open windows between 3 and 14 days, and level of evidence B in the USA and C in Europe (4-6). The risk of HT (and ICH) on an anticoagulant may be highest early, although it remains unknown and must be balanced against the benefit of reduction in recurrent ischemic strokes $(16,18)$. A focused guideline update in 2019 for stroke prevention in $\mathrm{AF}$ recommended DOACs over warfarin, excepting valvular disease (Class I, level of evidence A) (21), so AREST enrollment was suspended soon after this pronouncement. Regardless, as a randomized, controlled, multi-center trial, AREST will soon proffer some practical evidence concerning the safety and efficacy for early use of anticoagulation after AIS in AF patients while simultaneously balancing the inherent danger of AIS converting into $\mathrm{HT}$ as well as the risk of $\mathrm{CMB}$ development and progression into hemorrhagic strokes.

\section{ETHICS STATEMENT}

The studies involving human participants were reviewed and approved by University of South Florida Institutional Review Board/Institutional Ethics Committee. The patients/participants provided their written informed consent to participate in this study.

\section{AUTHOR CONTRIBUTIONS}

DR, JM, RM, BK, WB, and AL contributed to study conception, design, writing, and reviewing the manuscript. MF, SR, TK, AP, MM, RCM, AB, TM, NH, NW, and NT contributed to manuscript review. KK contributed to statistical aspects of the protocol.

\section{FUNDING}

The Bristol Myers Squibb/Pfizer Alliance provided support for this randomized study. 


\section{REFERENCES}

1. Wolf PA, Abbott RD, Kannel WB. Atrial fibrillation as an independent risk factor for stroke: the Framingham Study. Stroke. (1991) 22:983-8. doi: 10.1161/01.STR.22.8.983

2. Gage BF, Waterman AD, Shannon W, Boechler M, Rich MW, Radford MJ. Validation of clinical classification schemes for predicting stroke: results from the National Registry of Atrial Fibrillation. J Am Med Assoc. (2001) 285:2864-70. doi: 10.1001/jama.285.22.2864

3. Martin RC, Burgin WS, Schabath MB, Kirby B, Chae SH, Fradley MG, et al. Gender-specific differences for risk of disability and death in atrial fibrillation-related stroke. Am J Cardiol. (2017) 119:256-61. doi: 10.1016/j.amjcard.2016.09.049

4. Lansberg MG, O’Donnell MJ, Khatri P, Lang ES, Nguyen-Huynh MN, Schwartz NE, et al. Antithrombotic and thrombolytic therapy for ischemic stroke: Antithrombotic therapy and prevention of thrombosis, 9th ed.: American College of Chest Physicians Evidence-Based Clinical Practice Guidelines. Chest. (2012) 141:e601S-36S. doi: 10.1378/chest.141.4.1129b

5. Kernan WN, American Heart Association Stroke Council, Council on Cardiovascular and Stroke Nursing, Council on Clinical Cardiology, Council on Peripheral Vascular Disease, et al. Guidelines for the prevention of stroke in patients with stroke and transient ischemic attack: a guideline for healthcare professionals from the American Heart Association/American Stroke Association. Stroke. (2014) 45:2160-236. doi: 10.1161/STR.00000000000 00024

6. Kirchhof P, Benussi S, Kotecha D, Ahlsson A, Atar D, Casadei B, et al. ESC Scientific Document Group. 2016 ESC Guidelines for the management of atrial fibrillation developed in collaboration with EACTS. Eur Heart J. (2016) 37:2893-962. doi: 10.1093/eurheartj/ehw210

7. Gioia LC, Kate M, Sivakumar L, Hussain D, Kalashyan H, Buck B, et al. Early rivaroxaban use after cardioembolic stroke may not result in hemorrhagic transformation: a prospective magnetic resonance imaging study. Stroke. (2016) 47:1917-9. doi: 10.1161/STROKEAHA.116.013491

8. VISTA collaborators. Association of improved outcome in acute ischaemic stroke patients with atrial fibrillation who receive early antithrombotic therapy: analysis from VISTA. Eur J Neurol. (2015) 22:1048-55. doi: $10.1111 /$ ene. 12577

9. Paciaroni M, Agnelli G, Falocci N, Caso V, Becattini C, Marcheselli S, et al. Early recurrence and cerebral bleeding in patients with acute ischemic stroke and atrial fibrillation: effect of anticoagulation and its timing: the RAF Study. Stroke. (2015) 46:2175-82. doi: 10.1161/STROKEAHA.115.008891

10. Macha K, Volbers B, Bobinger T, Kurka N, Breuer L, Huttner HB, et al. Early initiation of anticoagulation with direct oral anticoagulants in patients after transient ischemic attack or ischemic stroke. J Stroke Cerebrovasc Dis. (2016) 25:2317-21. doi: 10.1016/j.jstrokecerebrovasdis.2016.06.031

11. Seiffge DJ, Traenka C, Polymeris A, Hert L, Peters N, Lyrer P, et al. Early start of DOAC after ischemic stroke: risk of intracranial hemorrhage and recurrent events. Neurology. (2016) 87:1856-62. doi: 10.1212/WNL.00000000000 03283

12. Rose DZ, Falcao D, Martin RC. Seek and ye shall find fibrillations. Stroke. (2016) 47:1969-71. doi: 10.1161/STROKEAHA.116.013987
13. Wardlaw JM, Dennis MS, Lindley RI, Sellar RJ, Warlow CP. The validity of a simple clinical classification of acute ischaemic stroke. J Neurol. (1996) 243:274-9. doi: 10.1007/BF00868526

14. Diener HC, Selim MH, Molina CA, Greenberg SM. Embolic stroke, atrial fibrillation, and microbleeds: is there a role for anticoagulation? Stroke. (2016) 47:904-7. doi: 10.1161/STROKEAHA.115.011150

15. Connolly SJ, Ezekowitz MD, Yusuf S, Eikelboom J, Oldgren J, Parekh A, et al. RE-LY Steering Committee and Investigators. Dabigatran versus warfarin in patients with atrial fibrillation. N Engl J Med. (2009) 361:1139-51. doi: 10.1056/NEJMoa0905561

16. Granger CB, Alexander JH, McMurray JJ, Lopes RD, Hylek EM, Hanna M, et al. Apixaban versus warfarin in patients with atrial fibrillation. N Engl J Med. (2011) 365:981-92. doi: 10.1056/NEJMoa1107039

17. Akase T, Tsuchiya T, Morita M. Hospitalization period and direct medical cost in patients using warfarin or novel oral anti-coagulants after a cerebral embolism. Int J Clin Pharm. (2019) 41:546-54. doi: 10.1007/s11096-019-00792-9

18. Patel MR, Mahaffey KW, Garg J, Pan G, Singer DE, Hacke W, et al. Rivaroxaban versus warfarin in nonvalvular atrial fibrillation. N Engl J Med. (2011) 365:883-91. doi: 10.1056/NEJMoa1009638

19. Giugliano RP, Ruff CT, Braunwald E, Murphy SA, Wiviott SD, Halperin JL, et al. Edoxaban versus warfarin in patients with atrial fibrillation. $N$ Engl J Med. (2013) 369:2093-104. doi: 10.1056/NEJMoa1310907

20. Cappellari M, Carletti M, Danese A, Bovi P. Early introduction of direct oral anticoagulants in cardioembolic stroke patients with nonvalvular atrial fibrillation. J Thromb Thrombolysis. (2016) 42:383-8. doi: 10.1007/s11239-016-1393-9

21. January CT, Wann LS, Calkins H, Chen LY, Cigarroa JE, Cleveland JC, et al. 2019 AHA/ACC/HRS Focused Update of the 2014 AHA/ACC/HRS Guideline for the Management of Patients With Atrial Fibrillation: A Report of the American College of Cardiology/American Heart Association Task Force on Clinical Practice Guidelines and the Heart Rhythm Society. Am J Cardiol. (2019) 74:104-32. doi: 10.1016/j.jacc.2019.01.011

Conflict of Interest Statement: DR receives modest honoraria for consulting or speaker bureau from Boehringer-Ingelheim and Medtronic as well as significant honoraria from Boston Scientific. MF receives modest honoraria for consulting from Novartis Pharmaceuticals.

The remaining authors declare that the research was conducted in the absence of any commercial or financial relationships that could be construed as a potential conflict of interest.

Copyright (C) 2019 Rose, Meriwether, Fradley, Renati, Martin, Kasprowicz, Patel, Mokin, Murtagh, Kip, Bozeman, McTigue, Hilker, Kirby, Wick, Tran, Burgin and Labovitz. This is an open-access article distributed under the terms of the Creative Commons Attribution License (CC BY). The use, distribution or reproduction in other forums is permitted, provided the original author(s) and the copyright owner(s) are credited and that the original publication in this journal is cited, in accordance with accepted academic practice. No use, distribution or reproduction is permitted which does not comply with these terms. 